Cristina Andenna, Klaus Herbers, Gert Melville (dir.), Die Ordnung der Kommunikation und die Kommunikation der Ordnungen, 1 : Netzwerke : Klöster und Orden im Europa des 12. und 13. Jahrhunderts / Cristina Andenna, Gordon Blennemann, Klaus Herbers, Gert Melville (dir.), Die Ordnung der Kommunikation und die Kommunikation der Ordnungen, 2 : Zentralität: Papsttum und Orden im Europa des 12. und 13. Jahrhunderts

Stuttgart : Steiner (Aurora - Schriften der Villa Vigoni, 1.1), 2012, 307 p., $56 €$ / Stuttgart : Steiner (Aurora - Schriften der Villa Vigoni, 1.2), 2013, 331 p., $56 €$

Ludovic Viallet

\title{
OpenEdition
}

\section{Édition électronique}

URL : http://journals.openedition.org/ifha/8080

DOI : 10.4000/ifha.8080

ISSN : 2198-8943

Éditeur

IFRA - Institut franco-allemand (sciences historiques et sociales)

Référence électronique

Ludovic Viallet, « Cristina Andenna, Klaus Herbers, Gert Melville (dir.), Die Ordnung der Kommunikation und die Kommunikation der Ordnungen, 1 : Netzwerke : Klöster und Orden im Europa des 12. und 13. Jahrhunderts / Cristina Andenna, Gordon Blennemann, Klaus Herbers, Gert Melville (dir.), Die Ordnung der Kommunikation und die Kommunikation der Ordnungen, 2 : Zentralität : Papsttum und Orden im Europa des 12. und 13. Jahrhunderts », Revue de l'IFHA [En ligne], Date de recension, mis en ligne le 14 avril 2015, consulté le 22 septembre 2020. URL : http://journals.openedition.org/ifha/8080 ; DOI : https:// doi.org/10.4000/ifha.8080 
Cristina Andenna, Klaus Herbers, Gert Melville (dir.), Die Ordnung der Kommunikation und die Kommunikation der Ordnungen, 1 : Netzwerke : Klöster und Orden im Europa des 12. und 13. Jahrhunderts / Cristina Andenna, Gordon Blennemann, Klaus Herbers, Gert Melville (dir.), Die Ordnung der Kommunikation und die Kommunikation der Ordnungen, 2 : Zentralität : Papsttum und Orden im Europa des 12. und 13. Jahrhunderts

Stuttgart : Steiner (Aurora - Schriften der Villa Vigoni, 1.1), 2012, 307 p., $56 €$ / Stuttgart : Steiner (Aurora - Schriften der Villa Vigoni, 1.2), 2013, 331 p., $56 €$

Ludovic Viallet

Ces deux ouvrages sont le fruit de rencontres organisées en novembre 2009 et juin 2010 au Centre italo-allemand pour l'excellence européenne de la Villa Vigoni (Loveno di 
Menaggio) par Gert Melville (fondateur de la FOVOG, Professeur émérite à la TU de Dresde) et Cristina Andenna (FOVOG, Université de la Basilicate, Matera/Potenza) avec Klaus Herbers (Université d'Erlangen-Nuremberg) et la collaboration de Gordon Blennemann (Erlangen-Nuremberg). Au cœur des travaux avait été placée l'étude des systèmes et structures de communication entre établissements réguliers et entre la papauté et les ordres religieux aux XIIe-XIIIe siècles, ainsi que l'idée d'une période décisive pour la formation d'un véritable espace culturel européen, dans les mots comme dans les pratiques. Ce bel ensemble de trente-deux textes, répartis équitablement entre les deux volumes, apporte donc une contribution importante à l'histoire des ordres religieux, de l'institution pontificale et de l'émergence progressive du concept d'Europe dans la matrice de la Chrétienté. Tout cela n'était pas gage d'unité et de cohérence, mais l'on se doit de souligner les efforts des coordinateurs pour organiser les contributions, offrir des résumés et des mises au point introductives sur certains concepts-clefs (celle du sociologue Alois Hahn sur les notions de "centre » et "périphérie » en tête du premier volume, l'introduction de K. Herbers, G. Melville et G. Blennemann puis la communication d'ouverture d'Agostino Paravicini-Bagliani «L'Europe est-elle un concept pour la papauté au Moyen Âge? » dans le second) et assurer un minimum d'articulation entre les deux ouvrages. À ce dernier objectif contribuent indubitablement, en clôture du premier volume, les synthèses intelligentes et complémentaires de Nicolangelo D'Acunto et Cristina Andenna sur la dialectique centre/périphérie au sein des ordres religieux - qu'ils aient été à centre "stable " (Cluny, Cîteaux, Prémontré, mais selon des modèles différents) ou à centre « mobile » et «fonctionnel » (Mendiants) - et leur capacité à communiquer à travers la réalisation de réseaux. Ces efforts ne suppriment toutefois pas la diversité thématique des contributions, orientées dans bien des directions; y parle-t-on toujours de " communication", d'ailleurs, et ce terme ne sert-il pas à beaucoup de choses? On laissera le lecteur juger. Le recenseur, lui, a une tâche déjà bien difficile en prétendant rendre compte d'une publication collective d'une telle ampleur.

Le volume Netzwerke: Klöster und Orden im Europa est organisé en quatre sections consacrées respectivement aux contenus (Inhalte), dispositifs (Einrichtungen), médias (Medien) et chemins (Wege) de la communication. La distribution des contributions est assez cohérente, avec, dans la première partie, des textes de Mirko Breitenstein sur la littérature parénétique, Jens Röhrkasten sur les données économiques et Florent Cygler sur les principaux traits de la communication au sein d'un ordre. Ce dernier auteur brosse une typologie des genres de la communication écrite et insiste sur le " haut degré de formalisation et de standardisation, c'est-à-dire en définitive de bureaucratisation" (p. 88) atteint par celle-ci au XIIIe siècle, le corollaire étant un formatage de l'information en partie contrebalancé par le rôle, insaisissable pour l'historien sinon par bribes et effets d'écho, de l'oralité et des contacts informels. Dans les « dispositifs » sont ensuite abordés les systèmes de contrôle (Maria Pia Alberzoni), les archives (Sébastien Barret) et les studia mendiants (Roberto Lambertini). On y trouvera d'intéressantes remarques, notamment sur le rôle des archives, longtemps négligées dans les textes normatifs des ordres, ou sur la constitution d'un corps de lectores dans des structures d'enseignement à usage interne visant à former et maintenir à niveau des frères impliqués dans les activités pastorales quotidiennes; on saisira aussi les limites de la distinction entre l'« horizontalité » de la communication au sein des ordres et la "verticalité » des relations de ceux-ci avec la papauté, donc l'intérêt d'appréhender Die Ordnung der Kommunikation comme un seul et même 
ensemble. En abordant les systèmes de contrôle qu'ont constitués le Chapitre général et la visite, M. P. Alberzoni souligne combien les dispositions de Latran IV, destinées à étendre le modèle cistercien à tous les monastères n'étant pas insérés dans un réseau, ont été difficilement applicables. L'étude, qui s'arrête particulièrement sur les cas dominicain et franciscain, est à rapprocher de celle de Hans-Joachim Schmidt dans le second volume, laquelle met en évidence les différences, voire les contradictions entre le modèle d'organisation et de communication voulu par le pouvoir pontifical et celui des Cisterciens, pourtant pris explicitement comme référence par le décret de Latran IV.

Dans la troisième section du volume, Uwe Israel aborde la question de la communication personnelle entre couvents à partir de la correspondance d'Hildegarde de Bingen et Wibert de Gembloux, en s'arrêtant en particulier sur le rôle du messager, sprechender Brief, auteur d'un véritable acte performatif; Giles Constable part des sources permettant de cerner la communication entre maisons religieuses (lettres, livres de confraternité et listes de défunts) pour souligner la place de l'amicitia et de son expression dans les écrits entre moines, Rudolf Kilian Weigand livre une réflexion dans l'optique de la Mediengeschichte - sur la parole du prédicateur (avant tout mendiant) et le livre jusqu'au XVe siècle ; enfin, Carola Jäggi tord le cou, s'il en était encore besoin, à l'idée d'un " plan-type » cistercien et d'une architecture exprimant la recherche d'uniformitas de l'Ordre et Heidrun Stein-Kecks part du texte de Boto de Prüfening (v. 1103 - v. 1170) De statu domus Dei pour effectuer un parallèle avec quatre peintures murales exécutées vers 1120 au sein de l'église abbatiale de Prüfening dans le contexte de la Querelle des Investitures et insister sur le rôle de l'image comme moyen de communication politique. Les messagers, la prédication, l'architecture, l'image... Des études de cas bien menées, mais aux conclusions en définitive assez attendues, et c'est peut-être dans la quatrième section, sur les « chemins » de la communication, que l'on trouvera les contributions les plus stimulantes, avec celles, déjà évoquées, de $\mathrm{N}$. D'Angelo et C. Andenna sur le forme della comunicazione negli ordini religiosi del XII e XIII secolo (N. D'Angelo, Il centro; C. Andenna, periferia e sottocentri) ainsi que l'étude plus spécifique qui les précède. Steven Vanderputten met en effet en évidence, dans la documentation issue du Comté de Flandre, l'apparition d'un discours de la «culpabilisation » qui s'accompagne chez les moines d'une insistance croissante sur la repentance et la pénitence comme moyen de réconciliation; de fait, un nombre significatif de chartes, entre les années 1080 et 1120-1130, font référence à des actes publics de contrition par des laïcs, en des gestes considérés comme ayant une signification politique. Ces chartes de règlement de conflits montrent comment, à la différence de la Provence étudiée par Florian Mazel ou du "paradigme » énoncé par Patrick Geary, les abbés du tournant des XIe-XIIe siècles se sont efforcés de normaliser leurs relations avec l'aristocratie locale et d'intégrer les seigneurs dans l'amicitia de leur communauté, marquant plus nettement une frontière entre les réguliers et la société laïque, affirmant ainsi de façon croissante que le but des deux groupes dans la société « était fondamentalement distinct et donc complémentaire » (p. 252).

Le volume Zentralität: Papsttum und Orden im Europa s'ouvre, après les deux contributions introductives, sur une section à texte unique, celui d'Uta Renate Blumenthal consacré aux décisions du concile de Latran III et à la pratique juridique qui en a résulté. Compte tenu du thème de l'ouvrage et de son économie globale, ce choix peut surprendre un peu, de même que l'insertion, en tête de la section suivante consacrée à la succession et à la protection de la communication 
(Kommunikationssukzession und -sicherung), de la synthèse de Jean-Marie Martin sur les transferts culturels entre monastères grecs et latins en Italie du Sud. Les textes qui s'enchaînent ensuite sont davantage dans le sujet: M. P. Alberzoni souligne bien, à partir du terrain privilégié de la région padane, comment les nouveaux groupes religieux (Umiliati, Poveri Cattolici) se sont tournés vers le Saint-Siège à partir de la seconde moitié du XIIe siècle afin de se protéger de l'accusation d'hérésie et comment ce dernier, avec Innocent III puis des légations de cardinaux comme Hugues d'Ostie, prônant les réformes de Latran IV, a utilisé les nouvelles communautés (en particulier mendiantes) comme points d'appui afin d'assurer l'essor de la prédication, le soutien à l'action pour la croisade et la pacification des cites; à la même époque, Honorius III (1216-1227) a développé l'organisation financière de la croisade et l'usage de la propagande en faveur de celle-ci, créant, selon Christian Grasso, un véritable « réseau informel et presque transversal à la réalité ecclésiastique, de spécialistes de la prédication de la croisade » (p.155); enfin, sur la longue durée des XIIe-XIIIe siècles, Waldemar Könighaus propose une synthèse sur les relations des papes avec les monastères d'Europe centro-orientale (Bohême, Hongrie, Pologne) et la progressive atténuation des différences existant, dans les structures ecclésiastiques, avec la Chrétienté occidentale. Ces contributions, comme les neuf des deux sections suivantes (Kommunikationsräume I: vom Zentrum zur Peripherie et Kommunikationsräume II: Zentralität und Hierarchie), traitent toutes des moyens avec lesquels la papauté a œuvré au déploiement de son autorité sur l'ensemble du monde chrétien européen : par la justice et les appels en Cour romaine (Patrick Zutshi), d'une façon générale l'affirmation du pape comme juge universel de la Chrétienté, donc des pratiques de communication que des ordres religieux comme les Cisterciens, Chartreux ou Prémontrés ont tâché de limiter afin qu'elles ne court-circuitent pas les procédures de leurs affaires internes (Guido Cariboni), le développement d'une juridiction déléguée n'étant d'ailleurs pas, à terme, sans nuire à l'exercice de l'autorité pontificale (Harald Müller); par l'idée de l'épiscopat universel du pape (Thomas Wetzstein) et des références - de moins en moins nombreuses - à la fonction de centralité de la Ville éternelle (Jochen Johrendt) ; par l'usage, en lien étroit avec l'apparition des Mendiants, de la canonisation (Roberto Paciocco) et la fonction, importante pour les ordres religieux, de cardinal protecteur, à laquelle $C$. Andenna consacre une étude novatrice avant de clore le volume, conjointement avec G. Blennemann, sur des propositions de mise en ordre conceptuelle (« Papsttum und Orden in der Interaktion: Beobachtungen und Perspektiven zur begrifflichen Einordnung »).

\section{INDEX}

Index chronologique : Moyen Âge

Thèmes : histoire religieuse 
AUTEUR

LUDOVIC VIALLET

Université Blaise Pascal, Clermont-Ferrand 2, CHEC 